\title{
A two-stage analysis of the effects of familiarization instructions on learning '
}

\author{
DEPARTMENT OF EDUCATION, THE JOHNS HOPKINS UNIVERSITY
}

This experiment investigate's the effects of familiarization instructions (Spell/Pronounce) on the two stages of a serial learning task. Significant differences in learning were found which favored the $\mathrm{P}$ instructions and which could not be accounted for in terms of differences in response acquisition. The results agree with the hypothesis that the facilitation which results from the use of $\mathrm{P}$ instructions extends to the associative stage of the serial learning task.

Previous research has disclosed that familiarization reduces associative latency (AL) but only when Ss are instructed to pronounce (P), not spell (S), the materials during familiarization training (Gillooly, 1965, 1966). Further, very little relevant familiarization (seven trials) with $P$ instructions (the PRF condition) was found to facilitate performance in a serial learning task (relative to irrelevantly familiarized controls using $P$ instructions-the PIF condition) whereas the same amount of relevant familiarization with $S$ instructions (SRF) did not produce facilitation over the respective controls (SIF) (1966). The locus of the effect of using $P$ instructions has yet to be identified in terms of the two stages of learning (Underwood \& Schulz, 1960).

Mechanic \& D'Andrea (1965) have interpreted their findings, that the free learning of a heterogeneous list composed of words and trigrams is aided more by $P$ than by $S$ instructions, as indicating that the letter sequence integration of response units is facilitated more by $P$ than by $S$ instructions. That is, their data lend support to the notion that familiarization exerts facilitation via the response-acquisition stage of learning and, consequently, any familiarization instructions which facilitate learning more than other instructions do so because the process of responseacquisition has been accelerated.

However, we cannot yet rule out the possibility that the PRF condition has a two-fold effect, that is, that effects are exerted via the associative stage in addition to whatever effects are exerted on the responseacquisition stage. The following facts were relevant to the development of such a notion: Familiarization has been shown to facilitate serial learning both with (Gillooly, 1966; the PRF condition) and without (Riley \& Phillips, 1959; using S-like instructions) concomitant changes in $\mathrm{AL}$. It seems plausible that facilitation which accompanies changes in $\mathrm{AL}$ would differ in kind from that which is not so accompanied. It is hypothesized that facilitation produced by $S$ instructions (and not attended by a decrease in $\mathrm{AL}$ ) is exerted via the response-acquisition stage alone, and the facilitation produced by $P$ instructions (attended by a decrease in $A L$ ) is exerted via both the associative and the response-acquisition stages of learning. That is, the effects of shorter ALs are thought to be exerted in the associative stage of a serial learning task.

This study reports the results of an initial investigation into this possibility.

Experimental Design

The experiment consisted of two parts: (1) relevant familiarization and (2) a serial learning task. There were two sets of instructions used in familiarization: spelling (for the Spell Relevant Familiarization condition, SRF) and pronouncing (for the Pronounce Relevant Familiarization condition, PRF). There were flve dependent variables, the first three of which presumably reflect the course of response-acquisition: the number of items correct in a free recall situation after familiarization; the number of items correctly recognized as coming from the familiarization task; the number of errors (overt as well as omissions) on the first anticipation trial of the learning task; the number of errors (overt plus omissions) to criterion in the learning task; and the number of trials to criterion in the learning task.

\section{Subjects}

Seventy-two male undergraduate students who were fulfilling a requirement in an introductory psychology course were randomly assigned to the two (Spell/ Pronounce) conditions ( $\mathrm{n} / 2$ per condition).

\section{Materials and Apparatus}

A 12 item list was constructed from the CVC trigrams forming Column 6 (22.7 average association value according to Glaze, 1928) in Hilgard's (1951) Table 7 (p. 540). The list was presented in the serial anticipation task by a Gerbrands memory drum.

\section{Procedure}

Familiarization. In individual sessions, Ss were given a pack of 723 in. $x 5$ in. cards on which were typed (one to a card in upper case letters) the task stimuli. Each stimulus was presented six times and the pack was shuffled after each S's use. In addition, Ss were given a six page booklet containing 72 lines (12 to a page) in which to write the trigrams. Ss were instructed either to (1) spell each trigram to himself three times while writing it (the Spell condition) or to (2) pronounce each trigram to himself three times while writing it (the Pronounce condition).

Immediately following the familiarization procedure 
there was a $5 \mathrm{~min}$ free recall period in which Ss wrote as many of the familiarized stimuli as they could (without forewarning from E). This, in turn, was followed by a recognition test for which Ss were given a sheet on which were typed (in upper case letters) the 72 trigrams in Hilgard's (1951) Table 7 with the items of Column 6 (the familiarized stimuli) distributed randomly throughout the remainder of the table. Ss were given $3 \mathrm{~min}$ to circle the previously familiarized items.

Leaming. In individual sessions immediately following the recognition test, Ss were required to learn (by serial anticipation) the 12 famillarized stimuli. The instructions required Ss of both familiarization conditions to respond by spelling the trigrams. The list was presented by a Gerbrands memory drum set as follows: 3 sec exposure per item, zero interitem interval, and $6 \mathrm{sec}$ intertrial interval. The criterion of mastery was two successive perfect recitations. The dependent variables were the number of trials and the number of errors to criterion as well as the number of errors committed on the first anticipation trial.

\section{Resulis and Discussion}

The difference between the SRF and PRF groups in the number of items recalled correctly from familiarization was not significant $(F<1.00$, df $=1 / 70)$ but favored the PRF group (6.91 items vs 6.67 for the SRF). Nor was the difference in the performance of the groups on the recognition task significant either $(F=1.79, \mathrm{df}=1 / 70, .10<\mathrm{p}<.20)$, although in favor of the PRF group (9.83 items vs 9.22 for the SRF). Since it is known that the number of correct responses on the first anticipation trial is a dependent variable sensitive to the differential effects of familiarization (Noble, 1955), these data were analyzed. The difference between the groups in the number of errors committed on the first anticipation trial was not significant $(F<1.00, d f=1 / 70)$ and in favor of the SRF group (10.3 v8 10.6 errors for the PRF). Apparently, there was no difference between the groups at this stage in the learning task.

Analysis of the learning data revealed that there was a significant difference in the performance of the groups on this task. In terms of the number of trials to criterion, the difference produced an $F=$ 4.75, which for $\mathrm{df}=1 / 70, \mathrm{p}<.05$ (the $P R F$ mean $=$ 21.1 trials and the SRF mean $=26.5$ trials). In terms of the number of errors to criterion, $F=4.60, \mathrm{df}=$ $1 / 70, p<.05$ (PRF mean $=99$ errors and the SRF mean $=$ 1.33 errors). The PRF group's learning has been facilitated to a significant extent.

In general, then, there was a difference between the PRF and SRF groups in the learning task which is unaccountable in terms of differences in the response-acquisition stage as measured by the three dependent variables employed in this study. This finding seems to diminish the likelihood that the PRF group superiority in learning is due to differences in the numbers of items recallable or recognizable, as measured by an unpaced procedure, after familiarization. This does not rule out the possibility, of course, that other differences in response availability may exist between the groups after familiarization. But the results, so far, are in consonance with the hypothesis that the effects of PRF may extend beyond the response-acquisition stage to the associative stage of the serial learning task.

\section{Reforences}

Gillooly, W. B. The effect of familiarization on associative latency. Psychon. Sci., 1965, 3, 235-236.

Gillooly, W. B. The effect of familiarization instructions on associative latency and learning. Psychon. Sci., 1966, 4, 303-304.

Glaze, J. A. The association value of non-sense syllables. $J$. genet. Psychol., 1928, 35, 255-267.

Hilgard, E. R. Methods and procedures in the study of learning. In S. S. Stevens (Ed.), Handbook of experimental psychology. New York: J. Wiley \& Sons, 1951.

Mechanic, A., \& D'Andrea, J. The role of articulation in response learning. Proc. 73rd Annu. Conv. Amer. Psychol. Assoc. Washington: Amer. Psychol. Assoc., 1965, 61-62.

Noble, C. E. The effect of familiarization upon serial verbal learning. J. exp. Psychol., 1955, 49, 333-338.

Riley, D. A., \& Phillips, L. W. The effects of syllable familiarization on rote learning, association value, and reminiscence. J. exp. Psychol., 1959, 57, 372-379.

Underwood, B. J., \& Schulz, R. W. Meaningfulness and verbal learning. Chicago: Lippincott, 1960.

\section{Notas}

1. The author wishes to express his appreciation to Lawrence $A$. Goldstein and Bruce $A$. Merhige who assisted by collecting the data.

2. Now at the Department of Educational Psychology, Rutgers University. 